\title{
Erratum: Vitamin E decreases bone mass by stimulating osteoclast fusion
}

Koji Fujita, Makiko Iwasaki, Hiroki Ochi, Toru Fukuda, Chengshan Ma, Takeshi Miyamoto, Kimitaka Takitani, Takako Negishi-Koga, Satoko Sunamura, Tatsuhiko Kodama, Hiroshi Takayanagi, Hiroshi Tamai, Shigeaki Kato, Hiroyuki Arai, Kenichi Shinomiya, Hiroshi Itoh, Atsushi Okawa \& Shu Takeda

Nat. Med. 18, 589-594 (2012); published online 4 March 2012; corrected after print 4 May 2012

In the version of this article initially published, it was incorrectly stated that the mice were fed a diet supplemented with a-tocopherol at 600 mg per $\mathrm{kg}$ of body weight. Instead, the food itself contained $600 \mathrm{mg}$ of $\alpha$-tocopherol per $\mathrm{kg}$. The error has been corrected in the HTML and PDF versions of the article.

\section{Corrigendum: Identification of a mutation in the extracellular domain of the Epidermal Growth Factor Receptor conferring cetuximab resistance in colorectal cancer}

Clara Montagut, Alba Dalmases, Beatriz Bellosillo, Marta Crespo, Silvia Pairet, Mar Iglesias, Marta Salido, Manuel Gallen, Scot Marsters, Siao Ping Tsai, André Minoche, Seshagiri Somasekar, Sergi Serrano, Heinz Himmelbauer, Joaquim Bellmunt, Ana Rovira, Jeff Settleman, Francesc Bosch \& Joan Albanell Nat. Med. 18, 221-223 (2012); published online 22 January 2012; corrected after print 6 July 2012

In the version of this article initially published, due to an oversight by the authors, the first and last names of one the authors, Somasekar Seshagiri, were incorrectly transposed. The error has been corrected in the HTML and PDF versions of the article.

\section{Corrigendum: Methylglyoxal modification of $\mathrm{Na}_{\mathrm{v}} 1.8$ facilitates nociceptive neuron firing and causes hyperalgesia in diabetic neuropathy}

\begin{abstract}
Angelika Bierhaus, Thomas Fleming, Stoyan Stoyanov, Andreas Leffler, Alexandru Babes, Cristian Neacsu, Susanne K Sauer, Mirjam Eberhardt, Martina Schnölzer, Felix Lasischka, Winfried L Neuhuber, Tatjana I Kichko, Ilze Konrade, Ralf Elvert, Walter Mier, Valdis Pirags, Ivan K Lukic, Michael Morcos, Thomas Dehmer, Naila Rabbani, Paul J Thornalley, Diane Edelstein, Carla Nau, Josephine Forbes, Per M Humpert, Markus Schwaninger, Dan Ziegler, David M Stern, Mark E Cooper, Uwe Haberkorn, Michael Brownlee, Peter W Reeh \& Peter P Nawroth

Nat. Med. 18, 926-933 (2012); published online 13 May 2012; corrected after print 6 July 2012
\end{abstract}

In the version of this article initially published, in Figure 1a, the bar for 3-deoxyglucosone was incorrectly labeled as 3-diacylglycerol. In Figure $6 \mathrm{~b}$, the labels for the control and methylglyoxal-treated samples were incorrectly switched. Also, Felix Lasitschka's last name was misspelled as Lasischka. The errors have been corrected in the HTML and PDF versions of the article.

\section{Corrigendum: Broad antigenic coverage induced by vaccination with virus-based cDNA libraries cures established tumors}

Timothy Kottke, Fiona Errington, Jose Pulido, Feorillo Galivo, Jill Thompson, Phonphimon Wongthida, Rosa Maria Diaz, Heung Chong, Elizabeth Ilett, John Chester, Hardev Pandha, Kevin Harrington, Peter Selby, Alan Melcher \& Richard Vile Nat. Med. 17, 854-859 (2011); published online 19 June 2011; corrected after print 14 August 2012

In the version of this article initially published, ASEL-CD8 in Figure $3 \mathrm{~h}$ was incorrectly labeled. The error has been corrected in the HTML and PDF versions of the article. 